\title{
Correction to: Efficient feature selection using shrinkage estimators
}

\author{
Konstantinos Sechidis ${ }^{1} \cdot$ Laura Azzimonti $^{2} \cdot$ Adam Pocock $^{3} \cdot$ Giorgio Corani $^{2}$. \\ James Weatherall ${ }^{4}$. Gavin Brown ${ }^{1}$
}

Published online: 4 June 2020

(c) The Author(s), under exclusive licence to Springer Science+Business Media LLC, part of Springer Nature 2020

\section{Correction to: Machine Learning (2019) 108:1261-1286 https://doi.org/10.1007/s10994-019-05795-1}

There was a mistake in the proof of the optimal shrinkage intensity for our estimator presented in Section 3.1. The main theorem still holds, and the shrinkage intensity presented in the corrected version is the optimal in the sense of minimizing the mean squared error (MSE). In this document, apart from correcting the proof for the optimal shrinkage intensity, we provide empirical verification on the correctness via simulations. The third term of Theorem 1 needs to be corrected as follows:

The original article can be found online at https://doi.org/10.1007/s10994-019-05795-1.

Konstantinos Sechidis

konstantinos.sechidis@manchester.ac.uk

Laura Azzimonti

laura@idsia.ch

Adam Pocock

adam.pocock@oracle.com

Giorgio Corani

giorgio@idsia.ch

James Weatherall

James.Weatherall@astrazeneca.com

Gavin Brown

gavin.brown@manchester.ac.uk

1 School of Computer Science, University of Manchester, Manchester, UK

2 Istituto Dalle Molle di studi sull' Intelligenza Artificiale (IDSIA), Manno, Switzerland

3 Oracle Labs, Burlington, MA, USA

4 Advanced Analytics Centre, Global Medicines Development, AstraZeneca, Cambridge, UK 


$$
\begin{aligned}
\widehat{\mathbb{E}}\left[\left(\hat{p}^{\mathrm{Ind}}(x y)\right)^{2}\right]=\frac{1}{N^{3}}( & (N-1)(N-2)(N-3)\left(\hat{p}^{\mathrm{ML}}(x) \hat{p}^{\mathrm{ML}}(y)\right)^{2} \\
& +(N-1)(N-2) \hat{p}^{\mathrm{ML}}(x) \hat{p}^{\mathrm{ML}}(y)\left(\left(\hat{p}^{\mathrm{ML}}(x)+\hat{p}^{\mathrm{ML}}(y)+4 \hat{p}^{\mathrm{ML}}(x y)\right)\right) \\
& +(N-1)\left(2 \hat{p}^{\mathrm{ML}}(x y)\left(\hat{p}^{\mathrm{ML}}(x)+\hat{p}^{\mathrm{ML}}(y)\right)+2\left(\hat{p}^{\mathrm{ML}}(x y)\right)^{2}\right. \\
& \left.\left.+\hat{p}^{\mathrm{ML}}(x) \hat{p}^{\mathrm{ML}}(y)\right)+\hat{p}^{\mathrm{ML}}(x y)\right) .
\end{aligned}
$$

Parts of supplementary material's pages $4-6$, where the above term is derived, need the following corrections. In page 4 the term $A(x y)$ needs to be corrected as follows:

$$
A(x y)=\sum_{\substack{x^{\prime}, x^{\prime \prime} \in \mathcal{X} \\ x^{\prime} \neq x^{\prime \prime} \neq x}} \sum_{\substack{y^{\prime}, y^{\prime \prime} \in \mathcal{Y} \\ y^{\prime} \neq y^{\prime \prime}}} \mathbb{E}\left[N_{x y^{\prime}} N_{x y^{\prime \prime}} N_{x^{\prime} y} N_{x^{\prime \prime} y}\right]+2 \sum_{\substack{x^{\prime} \in \mathcal{X} \\ x^{\prime} \neq x}} \sum_{\substack{y^{\prime}, y^{\prime \prime} \in \mathcal{Y} \\ y^{\prime} \neq y^{\prime \prime} \neq y}} \mathbb{E}\left[N_{x y^{\prime}} N_{x y^{\prime \prime}} N_{x^{\prime} y} N_{x y}\right] .
$$

As a consequence in page 5 the same term needs correction:

$$
\begin{aligned}
A(x y)= & N^{(4)}\left[\left(p(x)^{2}-\sum_{y^{\prime} \in \mathcal{Y}} p\left(x y^{\prime}\right)^{2}\right)\left(p(y)^{2}-\sum_{x^{\prime} \in \mathcal{X}} p\left(x^{\prime} y\right)^{2}\right)\right. \\
& \left.-4(p(x)-p(x y)) p(x y)^{2}(p(y)-p(x y))\right] .
\end{aligned}
$$

Finally, the first equation in page 6 needs the following correction:

$$
\begin{aligned}
& \sum_{x^{\prime}, x^{\prime \prime} \in \mathcal{X} y^{\prime}, y^{\prime \prime} \in \mathcal{Y}} \mathbb{E}\left[N_{x y^{\prime}} N_{x y^{\prime \prime}} N_{x^{\prime} y} N_{x^{\prime \prime} y}\right]=N^{(4)} p(x)^{2} p(y)^{2} \\
& \quad+N^{(3)} p(x) p(y)(p(x)+p(y)+4 p(x y)) \\
& +N^{(2)}\left[2 p(x y)(p(x)+p(y))+2 p(x y)^{2}+p(x) p(y)\right] \\
& \quad+N p(x y),
\end{aligned}
$$

which will result in the estimate for $\widehat{\mathbb{E}}\left[\left(\hat{p}^{\text {Ind }}(x y)\right)^{2}\right]$ presented in Eq. (1).

Apart from correcting the proof, we also provide some simulation results that validate the correctness of the optimal shrinkage intensity. To this end we followed the procedure described in the main paper's Section 3.2, to generate probabilities that lead to different types of effect size, i.e. different population values for the mutual information $I(X ; Y)$. The squared error of our shrinkage estimator for the probabilities is defined as $\sum_{x \in \mathcal{X}} \sum_{\mathcal{I}^{\prime} \mathcal{Y}}\left(p(x y)-\hat{p}^{\text {Ind-JS }}(x y)\right)^{2}$. We estimated the MSE by averaging over 1000 simulation runs. In Fig. 1 we present the results for three different effect sizes: $I(X ; Y)=0.01,0.05$ and 0.15 . In each graph we plot the MSE for all possible values of the shrinkage intensity $[0,1]$ and we also point out the optimal intensity using the corrected value $\lambda^{*}$ and the value we erroneously used in the previous version of the paper $\lambda_{e}^{*}$. As we see, the corrected value leads to the minimum MSE. 


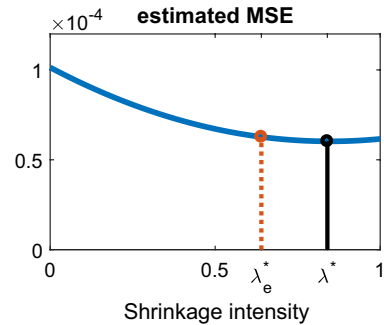

(a) $I(X ; Y)=0.01$

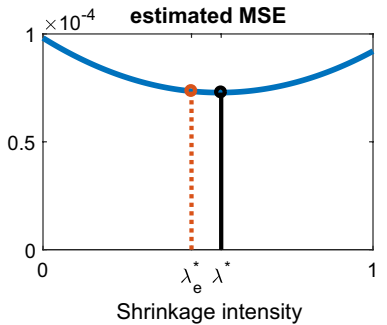

(b) $I(X ; Y)=0.05$

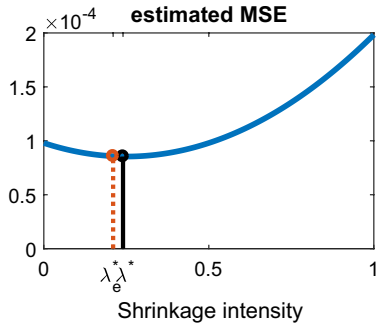

(c) $I(X ; Y)=0.15$

Fig. 1 Comparing the performance of our shrinkage estimator for different values of the shrinkage intensity in terms of MSE for three different effect sizes: a $I(X ; Y)=0.01$, b $I(X ; Y)=0.05$ and $\mathbf{c} I(X ; Y)=0.15$. The vertical lines show the optimal shrinkage intensity presented in this correction (solid black line), and the intensity erroneously presented in the initial version of the paper (dashed red line)

Acknowledgements We would like to thank Prof. Jan Mielniczuk and Małgorzata Łazęcka for bringing this issue to our attention and for their detailed and insightful comments.

Publisher's Note Springer Nature remains neutral with regard to jurisdictional claims in published maps and institutional affiliations. 